\title{
Pro-survival p53 target genes have evolved clusters of interacting polymorphic response elements that can affect cancer risk
}

Ping Zhang 1 , Giovanni Stracquadanio ${ }^{1}$, Xuting Wang2, Marc Pybus ${ }^{3}$, Jorge Zeron-Medina 3 , Svanhild Nornes ${ }^{1}$, Samantha Moore ${ }^{1}$, Ying Bi ${ }^{1}$, Marsha Wallace ${ }^{1}$, Elisabeth Bond ${ }^{1}$, Benjamin Davies ${ }^{4}$, Natalia Sacilotto ${ }^{1}$, Sarah De Val ${ }^{1}$, Benjamin Schuster-Boeckler ${ }^{1}$, Douglas Bell ${ }^{2}$, and Gareth Bond ${ }^{1}$

1. Ludwig Institute for Cancer Research, University of Oxford, Nuffield Department of Clinical Medicine, Old Road Campus Research Building, Oxford OX3 7DQ, UK

2. Environmental Genomics Group, Laboratory of Molecular Genetics, National Institute of Environmental Health Sciences-National Institutes of Health, Research Triangle Park, NC 27709, USA

3. Vall d'Hebron University Hospital, Oncology Department, Passeig de la Vall D'Hebron 119, 08035 Barcelona, Spain

4. Transgenic Technology Research Group, The Wellcome Trust Centre for Human Genetics, University of Oxford, Oxford OX3 7BN, UK

The p53 tumour suppressor is best known for its inhibition of cell survival through regulating transcription of anti-survival genes and $\sim 50 \%$ of cancers exhibit mutated p53, which promotes tumorigenesis. However, p53 also has prosurvival target genes and recent data from mice suggest that cancers retaining wild type p53 benefit from the activation of these genes. In humans, we find that pro-survival p53 target genes are more likely than anti-survival targets to have evolved clusters of polymorphic response elements that interact and affect cancer susceptibility. Using targeted genome editing, we evaluated a cluster of four genetically linked polymorphic p53 response elements and show that p53dependent up-regulation of a pro-survival gene can promote cancer cell survival through c-kit-mediated signaling. Our results provide human genetic evidence supporting a tumor-promoting role of pro-survival activities of p53. This urges re-thinking of p53's role in tumorigenesis and could support the development of more effective therapy combinations. 